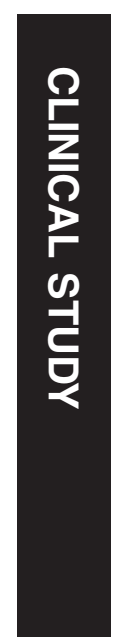

\section{Periodic acid-Schiff staining demonstrates fungi in chronic anterior blepharitis}

\begin{abstract}
Purpose To evaluate the presence of fungi in patients with chronic anterior blepharitis with periodic acid-Schiff (PAS) staining of the eyelashes in addition to the conventional methods of fungal cultures and direct microscopy.

Methods Nineteen patients with chronic anterior blepharitis of seborrheic or mixed seborrheic/staphylococcal type and $\mathbf{1 1}$ healthy age- and sex-matched controls were included in this prospective, nonrandomized, cross-sectional study. Blepharitis was diagnosed based on clinical evidence of greasy scales between the cilia, lid margin erythema, conjunctival hyperemia, telangiectasia, thickening, or irregularity of the eyelid margins by slit-lamp biomicroscopy. Eyelash samples were obtained by epilation with a sterile forceps and evaluated with PAS staining, fungal cultures, and direct microscopy. Results We demonstrated fungal elements with PAS staining in $\mathbf{7 9 \%}$ of the blepharitis group (hyphae and/or spores) and $18 \%$ of the control group. The difference was statistically significant $(P=0.002)$. Four patients in the blepharitis group (21\%) had positive cultures for fungi. The isolated fungi were Penicillium species ( 2 cases), Candida species ( 1 case), and Trichophyton verrucosum (1 case). Direct microscopic examination revealed Demodex mites in $42.1 \%$ of the blepharitis group. No culture growth or Demodex mites were observed in the control group. Conclusions We have shown fungi with PAS staining in the majority of patients with chronic anterior blepharitis. Further controlled studies are necessary to clarify the role of fungi in the etiopathogenesis of blepharitis.

Eye (2015) 29, 1522-1527; doi:10.1038/eye.2015.144; published online 21 August 2015
\end{abstract}

\section{Introduction}

Blepharitis is a frequently encountered clinical condition of eyelid margin inflammation that
Z Dadaci ${ }^{1}$, F Kılınç2 , TT Ozer ${ }^{3}$, GO Sahin ${ }^{4}$, NO Acir ${ }^{1}$ and M Borazan ${ }^{1}$ usually has a chronic course. Most common symptoms and signs are burning, stinging, itching, redness, flaking, and crusting of the eyelids. ${ }^{1}$ Chronic blepharitis is generally classified as anterior blepharitis, which involves the anterior lid margin and eyelashes, and posterior blepharitis, which involves meibomian glands. Anterior blepharitis is further divided into staphylococcal and seborrheic types, but usually mixed seborrheic/staphylococcal blepharitis is present. ${ }^{1,2}$

Chronic anterior blepharitis is one of the most common ocular disorders encountered in ophthalmological practice. Blepharitis is not a sight-threatening condition, but it may have a substantial impact on the patients' quality of life and, in its severe forms, it may lead to eyelid notching, scarring, trichiasis, loss of eyelashes, and corneal complications. ${ }^{1}$ Diagnosis is usually based on signs, symptoms, and the clinical appearance of the eyelids. ${ }^{2,3}$ Although the exact etiopathogenesis is unknown, many researchers have discussed bacteria, especially

Staphylococcus, Corynebacterium, and

Propronibacterium species, and Demodex mites as a cause. $^{2-4}$ In addition, chronic blepharitis is related with some inflammatory skin conditions such as seborrheic dermatitis and rosacea. ${ }^{5}$ Besides the discrepancy in its etiology, the treatment of chronic blepharitis is also controversial, and total cure is not possible in most cases. ${ }^{5,6}$

Fungi are rarely taken into account among the causes of anterior blepharitis. Culture is the gold standard for demonstration of fungi in tissues, but it may yield low-positive results. ${ }^{7,8}$ Periodic acid-Schiff (PAS) staining is another frequently used and reliable method for identification of fungi in tissues that may be superior to culture because demonstration of fungi in cultures may not be always possible. ${ }^{8}$

In our study, we report PAS staining, fungal culture, and direct microscopy results of 
eyelashes pulled from patients with clinical seborrheic blepharitis or mixed seborrheic/staphylococcal blepharitis and compared with healthy controls. We did not obtain bacterial cultures for staphylococci because these bacteria are also commonly present in normal ocular flora. 3,9 To the best of our knowledge, there are no previous reports of PAS staining of pulled eyelashes for detection of fungi in chronic blepharitis.

\section{Materials and methods}

\section{Study population}

This prospective, nonrandomized, cross-sectional study comprised 19 adult patients with chronic anterior blepharitis of seborrheic or mixed seborrheic/ staphylococcal type who had signs and symptoms of moderate to severe blepharitis for at least 2 years and 11 age- and sex-matched healthy controls. Blepharitis was diagnosed based on clinical evidence of greasy scales between the cilia, lid margin erythema, conjunctival hyperemia, telangiectasia, thickening, or irregularity of the eyelid margins. All patients had symptoms of itching, burning sensation, redness, and scaling in the eyelid margin and had several periods of treatment previously with no long-lasting relief. None of the patients in the blepharitis group had ulcerative blepharitis or meibomian gland disease. All subjects were recruited between April and August 2014, and evaluated at the Mevlana University eye clinic. The study protocol was approved by the Mevlana University ethics committee (2014/034). The study was performed in compliance with good clinical practice guidelines and in accordance with the principles of the Declaration of Helsinki. Written informed consent was obtained from each patient after explanation of the purpose and process of the study.

Pregnant or breastfeeding women, contact lens wearers, and patients who used antifungals within 4 weeks before entry into the study were excluded. None of the patients received treatment for blepharitis within 3 months before sampling. However, all patients had several periods of treatment, including topical antibiotics, steroids, and artificial tears, previously. Two subjects in the blepharitis group were using antiglaucomatous eye drops ( $\beta$-blockers). Subjects in the control group had normal ocular examination except refractive disorders within \pm 3.0 diopters and were not using any eye drops. Patients with any ocular pathology such as dry eye disease, pterygium, and allergic conjunctivitis were excluded. All participants underwent a comprehensive ophthalmological examination including best-corrected visual acuity obtained with a Snellen projection chart, refraction, slit-lamp biomicroscopy, and fundus examination.

\section{Sample collection}

Eyelash samples were obtained by epilation of eight eyelashes (four eyelashes from each eye) with a sterile forceps. We paid attention to epilate the eyelashes with their roots. We inoculated the first four eyelashes into two separate Sabouraud's dextrose agar (SDA) media for fungal cultures. Media were incubated at 25 and $37^{\circ} \mathrm{C}$ for a minimum period of 4 weeks. The growths were noted for colony characteristics in the form of rate and texture of growth, surface color, and color on reverse and diffusible pigments. For microscopic morphology, cellophane tape mounts with lactophenol cotton blue were done. Yeastlike cells or blastoconidia were searched on SDA colonies that are white to cream colored, smooth, glabrous, and yeast-like in appearance. Spherical to subspherical budding, yeast-like cells or blastoconidia were also stained with Gram stain. Germ tube test was applied to define Candida albicans. To perform germ tube test, we lightly inoculated $5 \mathrm{ml}$ of serum, containing $0.5 \%$ glucose and incubated at $35^{\circ} \mathrm{C}$ for $2-3 \mathrm{~h}$.

For direct microscopy, we put two eyelashes on a slide and added one drop of $15 \%$ potassium hydroxide $(\mathrm{KOH})$. A microscopic examination was carried for the presence of fungal elements after incubating the slides for 15-20 min or until digestion of specimens occurred.

For pathologic examination, two eyelashes were fixed in $96 \%$ alcohol and stained by PAS without prior sectioning. For PAS staining, the samples were incubated in $0.1 \%$ periodic acid for $10 \mathrm{~min}$. The eyelashes were washed in running tap water for $1 \mathrm{~min}$ and immersed in Schiff's reagent for $17 \mathrm{~min}$. Subsequently, the samples were washed in tap water for $3 \mathrm{~min}$, counterstained with Mayer's hematoxylin for $2 \mathrm{~min}$, washed in tap water for 3 min, and dehydrated in two changes of $96 \%$ alcohol. Finally, the samples were cleared in xylene and mounted with Entellan (Shandon Consul-Mount; Richard-Allan Scientific, Kalamazoo, MI, USA).

Both pathologist and microbiologist were blind to the group of the specimens.

\section{Statistical analysis}

Statistical analyses were performed using SPSS version 15.0 (SPSS Science, Chicago, IL, USA). Mean age of groups were compared with Mann-Whitney $U$-test. Distribution of sex, PAS staining, and direct microscopy results between the groups were compared using the $\chi^{2}$ test. The $P$-values of $<0.05$ were considered as statistically significant.

\section{Results}

A total of 19 patients (11 male and 8 female) with seborrheic blepharitis or mixed seborrheic/staphylococcal 
a
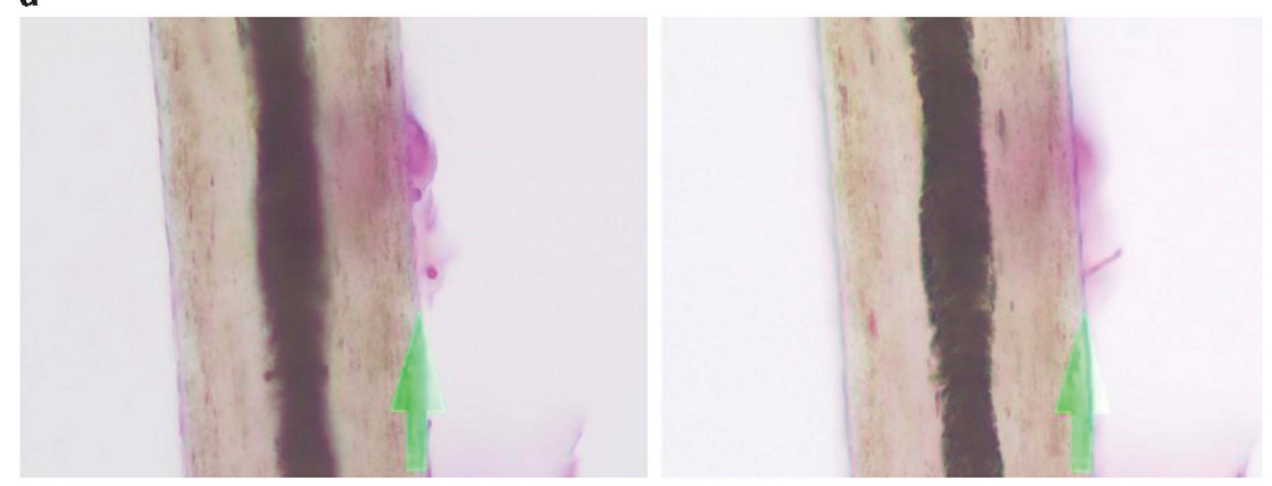

b

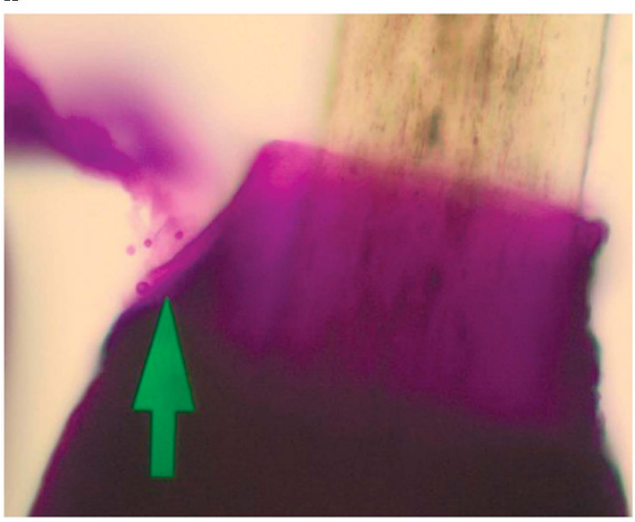

Figure 1 Periodic acid-Schiff staining. Appearance of spores and hyphae over the eyelash from case 1 (a) and appearance of spores near the root of the eyelash (b) from case 10 (original magnification $\times 400$ ).

blepharitis and 11 healthy controls ( 6 male and 5 female) were enrolled in our study. The mean ages of patients with blepharitis and control subjects were $50.74 \pm 20.93$ years (median 58 , range $18-80$ years) and $55.73 \pm 17.33$ years (median 58 , range $27-80$ years), respectively. No significant differences were found between the groups with respect to age ( $P=0.59$, Mann-Whitney $U$-test) and $\operatorname{sex}\left(P=1.00, \chi^{2}\right.$ test $)$.

We demonstrated fungal elements with PAS staining (Figure 1) in 79\% of the blepharitis group (hyphae and/or spores) and $18 \%$ of the control group. The difference was statistically significant $\left(P=0.002, \chi^{2}\right.$ test). In subjects with positive PAS staining, we found minimum 2 and maximum 75 spores on two eyelashes. In the blepharitis group, 4 patients had $<5$ spores, 5 patients had $5-10$ spores, and 6 patients had $>10$ spores. Two subjects with positive PAS staining in the control group had 2 and 8 spores, respectively. Four patients in the blepharitis group $(21 \%)$ had positive cultures for fungi. The isolated fungi were Penicillium species (2 cases), Candida species (1 case), and Trichophyton verrucosum (1 case). Direct microscopic examination revealed fungal elements in 11 cases $(58 \%)$ and demodex mites (3-4 mites per epilated lash) in 8 cases

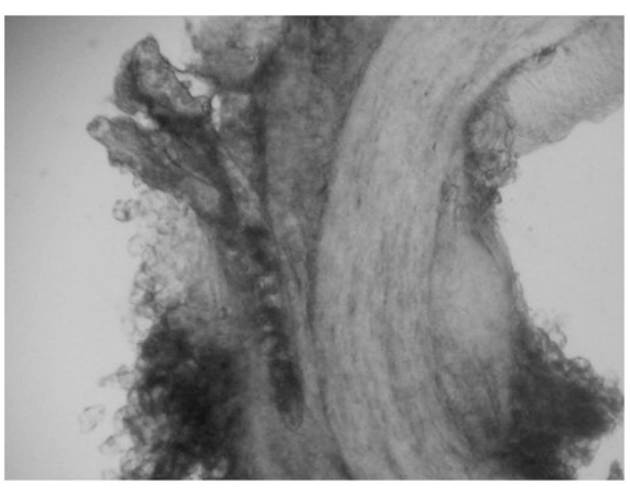

Figure 2 Appearance of Demodex mites on the direct microscopy of the pulled eyelash from case 11 at $1 \mathrm{~h}$ after application of $15 \%$ potassium hydroxide. A group of Demodex mites are seen in the upper left part of the figure (original magnification $\times 160$ ).

(42\%; Figure 2). The data of all patients in the blepharitis group are shown in Table 1. We detected fungal elements by direct microscopy in one subject (9\%) in the control group who also had positive PAS staining. No culture growth or Demodex mites on direct microscopic examination was observed in the control 
Table 1 Data of all patients in the chronic anterior blepharitis group

\begin{tabular}{|c|c|c|c|c|c|c|}
\hline Patient number & Gender & Age (years) & $\begin{array}{c}\text { Number of spores in } \\
\text { PAS stain }\end{array}$ & Culture & $\begin{array}{l}\text { Fungal elements on } \\
\text { direct microscopy }\end{array}$ & $\begin{array}{c}\text { Demodex on direct } \\
\text { microscopy }\end{array}$ \\
\hline 1 & M & 70 & $5-10$ & - & + & - \\
\hline 2 & M & 30 & $5-10$ & + & + & + \\
\hline 3 & $\mathrm{M}$ & 73 & $<5$ & - & + & + \\
\hline 4 & M & 65 & $<5$ & - & + & + \\
\hline 5 & M & 68 & 5-10 & - & + & + \\
\hline 6 & $\mathrm{~F}$ & 58 & - & - & - & - \\
\hline 7 & $\mathrm{~F}$ & 18 & - & - & - & - \\
\hline 8 & $\mathrm{~F}$ & 80 & $>10$ & - & - & - \\
\hline 9 & $\mathrm{~F}$ & 27 & - & - & - & - \\
\hline 10 & M & 22 & $5-10$ & - & + & + \\
\hline 11 & M & 69 & $>50$ & + & + & + \\
\hline 12 & $\mathrm{M}$ & 69 & $>10$ & + & + & + \\
\hline 13 & M & 67 & 5-10 & + & - & - \\
\hline 14 & $\mathrm{~F}$ & 61 & $>10$ & - & - & - \\
\hline 15 & $\mathrm{~F}$ & 31 & - & - & - & - \\
\hline 16 & M & 51 & $>50$ & - & + & + \\
\hline 17 & $\mathrm{~F}$ & 27 & $>10$ & - & - & - \\
\hline 18 & $\mathrm{~F}$ & 24 & $<5$ & - & + & - \\
\hline 19 & M & 54 & $<5$ & - & + & - \\
\hline
\end{tabular}

Abbreviations: M, male; F, female; PAS, periodic acid-Schiff.

Table 2 Comparison of the positive cases between the groups

\begin{tabular}{lcc}
\hline & $\begin{array}{c}\text { Blepharitis } \\
\text { group } \\
(\mathrm{n}=19)\end{array}$ & $\begin{array}{c}\text { Control } \\
\text { group } \\
(\mathrm{n}=11)\end{array}$ \\
\hline Periodic acid-Schiff stain & 15 & $2^{*}$ \\
Culture & 4 & - \\
Fungal elements on direct microscopy & 11 & $1^{+}$ \\
Demodex on direct microscopy & 8 & - \\
\hline
\end{tabular}

$P$-value: ${ }^{*} 0.002,{ }^{\dagger} 0.018$ ( $\chi^{2}$ test).

group. The comparison of the results between the groups is presented in Table 2 .

\section{Discussion}

This is a preliminary study demonstrating the presence of fungi with PAS staining of the eyelashes in patients with chronic anterior blepharitis.

Anterior blepharitis is an inflammatory disorder of the lid border that is usually considered as an infectious disease. To date, mostly bacteria and demodex mites have been discussed as causes of blepharitis. Staphylococcus, Propionibacterium, and Corynebacterium have been identified as major isolates from the lids of patients with blepharitis in previous reports, $, 2,10$ but these bacteria are also reported to be present in the normal ocular flora. ${ }^{9,11}$ In more recent studies, the difference in the relative proportions of these bacteria is indicated to have role in the pathogenesis of blepharitis. Lee et al ${ }^{11}$ reported lower proportions of Propionibacterium in patients with blepharitis and concluded that the balance between ocular microbiota may have a role in the ocular health. This might also explain our results as it is known that the decrease of normal microbial flora results in accumulation of pathogen organisms including fungi. ${ }^{12}$

Fungi are rarely proposed as a cause of chronic anterior blepharitis. More than half a century ago, it was reported that yeasts may play a role in the pathogenesis of both seborrheic dermatitis and blepharitis. ${ }^{13}$ Since then, there are few reports in the literature demonstrating fungi as a cause of chronic blepharitis. ${ }^{14-16}$ We were able to find only one clinical trial in our search of the literature, in which antifungal treatment of patients with seborrheic blepharitis yielded better results when compared with the conventional therapy. ${ }^{17}$

We demonstrated fungi in $78.9 \%$ of the patients with chronic anterior blepharitis with PAS staining of the eyelashes, significantly higher than the control group $(P=0.002)$. All the patients in the blepharitis group had signs and symptoms of blepharitis for $>2$ years, with several periods of treatments with no long-lasting success. Fungi may be the primary pathogens that would readily explain this lack of long-term cure. Alternatively, longterm use of antibiotics and/or steroids in the chronic clinical course of the disease might have increased the frequency fungi that may add to the chronic clinical course of the disease and unresponsiveness to the conventional therapy. It is known that fungi may become virulent in immune compromised situations such as long-term corticosteroid use. ${ }^{18}$ We have isolated 
T. verrucosum, Candida species, and Penicillium species in a total of four patients. These fungi had been reported as pathogens in various fungal ocular infections in previous reports. ${ }^{14,19-21}$ Candida species and T. verrucosum were reported in chronic blepharitis, ${ }^{14-16}$ but, to the best of our knowledge, there are no previous reports demonstrating Penicillium species in patients with chronic blepharitis.

When we graded our findings according to the number of spores counted on PAS-stained eyelashes, $40 \%$ (6 out of 15) of the patients with blepharitis had $>10$ spores, and 2 among them had $>50$ spores. As we included patients with signs and symptoms of moderate to severe blepharitis and obtained only two eyelashes for PAS staining, we could not make a comparison between the groups. But our findings would still pave the way for future clinical studies with larger number of patients correlating the severity of disease with the degree of fungal colonization.

We observed Demodex mites in $42.1 \%$ of patients with chronic blepharitis but none in the control group.

Although Demodex mites are reported more frequently in patients with blepharitis when compared with normal controls, there is controversy about their being a cause of blepharitis. ${ }^{22,23}$ Kim et al ${ }^{23}$ demonstrated significantly higher levels of IL-17 in the tears of Demodex blepharitis patients when compared with Demodex-free blepharitis group that normalized after treatment. They concluded that this finding may explain the mechanism of ocular discomfort and telangiectasias that is frequently encountered in Demodex blepharitis. In the blepharitis group, we have also demonstrated fungi by PAS staining in $78.9 \%$ of patients, significantly higher than the control group $(P=0.002)$. These results suggested that fungi and/ or Demodex mites may be the primary pathogens in chronic anterior blepharitis, or other factors such as increased seborrhea may facilitate the growth of these organisms ${ }^{24,25}$ that in turn might add to the signs and symptoms of blepharitis through inducing inflammation.

Our study indicates that fungi may be more common in chronic blepharitis than it was thought before. Mainly fungal cultures were used for investigation of fungi in blepharitis in previous studies, ${ }^{15,16}$ but fungal cultures may have a high false negative ratio because of both specific growth requirements of fungi and the usually limited size of available samples. In a series of 30 eyes with presumed fungal keratitis, positive fungal culture rates were $40 \%$, whereas PCR showed positivity in $93.3 \%$ of cases. ${ }^{7}$ In a recent report describing a series of five eyes removed for fungal endophthalmitis that were all positive for Bipolaris hawaiiensis by genotyping, three were culture negative. However, all eyes demonstrated hyphal and spore forms with fungal stains. The authors concluded that PAS staining of specimens obtained by vitrectomy was the most rapid and effective proof that fungi were present. $^{8}$
Ours was a preliminary study demonstrating fungi with PAS staining in chronic anterior blepharitis, but it has some limitations that should be addressed. The first limitation is the low number of the study participants, especially in the control group. However, there are several studies evaluating blepharitis in the literature that also have small case numbers. ${ }^{9,11,23}$ Another reason was our unwillingness in pulling eyelashes that may be painful from healthy subjects without any ocular symptoms. Finally, PCR might also be used for the demonstration of fungi, but this would increase the cost of the study. Still, this would be the subject of a further study as we wanted to emphasize PAS staining in the present study.

In conclusion, we have demonstrated fungi with PAS staining in the majority of the subjects in the chronic blepharitis group. This finding may be related with the alteration of normal ocular flora in chronic blepharitis and colonization of the eyelids by pathogen organisms including fungi that may further exacerbate the disease by causing inflammation. PAS staining is an easy, rapid, and inexpensive method of demonstrating fungi in patients with blepharitis. To reveal the role of fungi in chronic anterior blepharitis, larger controlled studies should be performed in the future. Demonstration of fungi as an etiologic factor in further larger-scale studies may change the therapeutic approach to chronic blepharitis that might include application of diluted antifungal shampoo for lid hygiene.

\section{Summary}

What was known before

- Exact etiology of chronic anterior blepharitis is unknown.

- Usually bacteria are blamed.

- Diluted baby shampoo, antibiotics, and weak steroids are used for treatment.

What this study adds

- Fungi may play a role in the pathogenesis of chronic anterior blepharitis, especially in cases unresponsive to classic treatment.

- Further investigations and randomized controlled trials are necessary to investigate the role of antifungals in the treatment of chronic anterior blepharitis.

\section{Conflict of interest}

The authors declare no conflict of interest.

\section{References}

1 Kanski JJ. Clinical Ophthalmology. Reed Educational and Professional Publishing Ltd: Oxford, UK, 1999, pp 8-12. 
2 Dougherty JM, McCulley JP. Comparative bacteriology of chronic blepharitis. Br J Ophthalmol 1984; 68(8): 524-528.

3 Groden LR, Murphy B, Rodnite J, Genvert GI. Lid flora in blepharitis. Cornea 1991; 10(1): 50-53.

4 Liu J, Sheha H, Tseng SCG. Pathogenic role of Demodex mites in blepharitis. Curr Opin Allergy Clin Immunol 2010; 10 (5): 505-510.

5 Bernardes TF, Bonfioli AA. Blepharitis. Semin Ophthalmol 2010; 25(3): 79-83.

6 Lindsley K, Matsumura S, Hatef E, Akpek EK. Interventions for chronic blepharitis. Cochrane Database Syst Rev 2012; 5: CD005556.

7 Tananuvat N, Salakthuantee K, Vanittanakom N, Pongpom M, Ausayakhun S. Prospective comparison between conventional microbial work-up vs PCR in the diagnosis of fungal keratitis. Eye (Lond) 2012; 26(10): 1337-1343.

8 Minckler D, Small KW, Walsh TJ. Clinical and pathologic features of Bipolaris endophthalmitis after intravitreal triamcinolone. JAMA Ophthalmol 2014; 132(5): 630-632.

9 Oto S, Aydin P, Ciftçioglu N, Dursun D. Slime production by coagulase-negative staphylococci isolated in chronic blepharitis. Eur J Ophthalmol 1998; 8(1): 1-3.

10 Ta CN, Shine WE, McCulley JP, Pandya A, Trattler W, Norbury JW. Effects of minocycline on the ocular flora of patients with acne rosacea or seborrheic blepharitis. Cornea 2003; 22(6): 545-548.

11 Lee SH, Oh DH, Jung JY, Kim JC, Jeon CO. Comparative ocular microbial communities in humans with and without blepharitis. Invest Ophthalmol Vis Sci 2012; 53(9): 5585-5593.

12 Wang Z, Xiao G, Yao Y, Guo S, Lu K, Sheng Z. The role of bifidobacteria in gut barrier function after thermal injury in rats. J Trauma 2006; 61(3): 650-657.

13 Thygeson P, Vaughan DG Jr. Seborrheic blepharitis. Trans Am Ophthalmol Soc 1954; 52: 173-188.

14 Sahin GO, Dadaci Z, Ozer TT. Two cases of tinea ciliaris with blepharitis due to Microsporum audouinii and Trichophyton verrucosum and review of the literature. Mycoses 2014; 57(9): 577-580.

15 Karimian F, Zarei-Ghanavati S, A BR, Jadidi K, Lotfi-Kian A. Microbiological evaluation of chronic blepharitis among Iranian veterans exposed to mustard gas: a case-controlled study. Cornea 2011; 30(6): 620-623.

16 Huber-Spitzy V, Böhler-Sommeregger K, Arocker-Mettinger E, Grabner G. Ulcerative blepharitis in atopic patients-is Candida species the causative agent? Br J Ophthalmol 1992; 76(5): 272-274.

17 Nelson ME, Midgley G, Blatchford NR. Ketoconazole in the treatment of blepharitis. Eye (Lond) 1990; 4(Pt 1): 151-159.

18 Henry CR, Flynn Jr HW, Miller D, Forster RK, Alfonso EC. Infectious keratitis progressing to endophthalmitis: a 15-year study of microbiology, associated factors, and clinical outcomes. Ophthalmology 2012; 119(12): 2443-2449.

19 Xie L, Zhai H, Shi W, Zhao J, Sun S, Zang X. Hyphal growth patterns and recurrence of fungal keratitis after lamellar keratoplasty. Ophthalmology 2008; 115(6): 983-987.

20 Nath R, Baruah S, Saikia L, Devi B, Borthakur AK, Mahanta J. Mycotic corneal ulcers in upper Assam. Indian J Ophthalmol 2011; 59(5): 367-371.

21 Shyong MP, Chen SJ, Lee FL, Hsu WM, Liu JH. Pseudophakic Penicillium endophthalmitis. Zhonghua Yi Xue Za Zhi (Taipei) 2000; 63(10): 770-773.

22 Hay R. Demodex and skin infection: fact or fiction. Curr Opin Infect Dis 2010; 23(2): 103-105.

23 Kim JT, Lee SH, Chun YS, Kim JC. Tear cytokines and chemokines in patients with Demodex blepharitis. Cytokine 2011; 53(1): 94-99.

24 Holzchuh FG, Hida RY, Moscovici BK, Villa Albers MB, Santo RM, Kara-José N et al. Clinical treatment of ocular Demodex folliculorum by systemic ivermectin. Am J Ophthalmol 2011; 151(6): 1030-1034.e1.

25 Zisova LG. Malassezia species and seborrheic dermatitis. Folia Med (Plovdiv) 2009; 51(1): 23-33. 